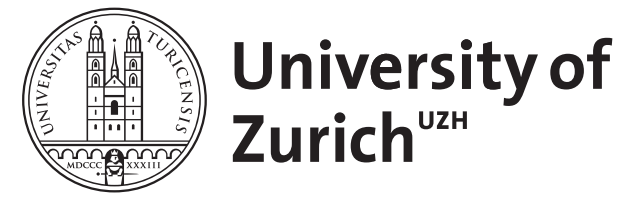

\title{
Absolute configuration of (S)-(+)-[(Benzyloxymethyl)(tert-butyl)methylsilyl] methyl ketone
}

\author{
Bratovanov, S ; Linden, Anthony ; Bienz, Stefan
}

\begin{abstract}
The absolute configuration of the title compound was confirmed by an X-ray crystal structure analysis of (R)-(+)-[(benzyloxymethyl)(tert-butyl)methylsilyl] methyl ketone 2,4,6-triisopropylphenylsulfonylhydrazone, C30H48N203SSi, reported herein. The asymmetric unit of the hydrazone contains two molecules which are linked by intermolecular hydrogen bonds.
\end{abstract}

DOI: https://doi.org/10.1107/S0108270195014338

Posted at the Zurich Open Repository and Archive, University of Zurich ZORA URL: https://doi.org/10.5167/uzh-138901

Journal Article

Published Version

Originally published at:

Bratovanov, S; Linden, Anthony; Bienz, Stefan (1996). Absolute configuration of (S)-(+)-[(Benzyloxymethyl)(tertbutyl)methylsilyl] methyl ketone. Acta Crystallographica. Section C: Crystal Structure Communications, 52(4):1045-1047.

DOI: https://doi.org/10.1107/S0108270195014338 
Acta Cryst. (1996). C52, 1045-1047

\section{Absolute Configuration of (S)-(+)-[(Benzyl- oxymethyl)(tert-butyl)methylsilyl] Methyl} Ketone

Svetoslav Bratovanov, Anthony Linden* and Stefan BIENZ

Organisch-chemisches Institut, Universität Zürich, Winterthurerstrasse 190, CH-8057 Zürich, Switzerland. E-mail: alinden@oci.unizh.ch

(Received 20 September 1995; accepted 16 October 1995)

\section{Abstract}

The absolute configuration of the title compound was confirmed by an X-ray crystal structure analysis of $(R)-(+)-[($ benzyloxymethyl)(tert-butyl)methylsilyl] methyl ketone 2,4,6-triisopropylphenylsulfonylhydrazone, $\mathrm{C}_{30} \mathrm{H}_{48} \mathrm{~N}_{2} \mathrm{O}_{3} \mathrm{SSi}$, reported herein. The asymmetric unit of the hydrazone contains two molecules which are linked by intermolecular hydrogen bonds.

\section{Comment}

We have recently demonstrated that chiral acylsilanes of type (I) can be efficiently used as starting materials for the stereoselective preparation of $\alpha$-hydroxysilanes (Bienz \& Chapeaurouge, 1991; Chapeaurouge \& Bienz, 1993). The latter compounds can be transformed into silicon-free secondary alcohols (Bienz et al., 1995) or they can be used as starting materials for other stereospecific transformations (Enev, Stojanova \& Bienz, 1995).<smiles>CC(=O)[Si](C)(Br)COc1ccccc1</smiles>

$(S)-(+)-(I)$

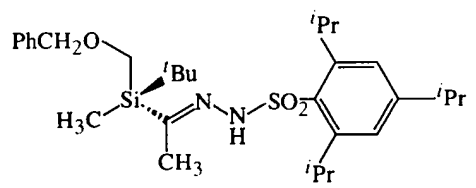

$(R)-(+)-(I I)$
Optically active $(S)-(+)-[($ benzyloxymethyl)(tert-butyl)methylsilyl] methyl ketone, $(S)-(+)-(\mathrm{I})$, and its optical antipode are accessible by a route involving a bioreduction step (Bienz et al., 1995). The absolute configuration at the chiral silicon centre in each of these compounds was determined by a combination of the empirical Mosher method and Nuclear Overhauser Effect (NOE) experiments. However, the NOE effects upon which the assignments are based are rather weak. Therefore, in order to secure unambiguously the absolute configuration of $(S)-(+)-(\mathrm{I})$, the X-ray structure of its hydrazone derivative, $(R)-(+)-(\mathrm{II})$, was determined.
The asymmetric unit of (II) contains two molecules, $A$ and $B$, of the same enantiomorph. Views of molecules $A$ and $B$, showing the atomic numbering and the correct absolute configuration, are given in Fig. 1. The absolute configuration was determined by refinement of the structure and the absolute-structure parameter (Flack, 1983), as described in the Experimental. Both

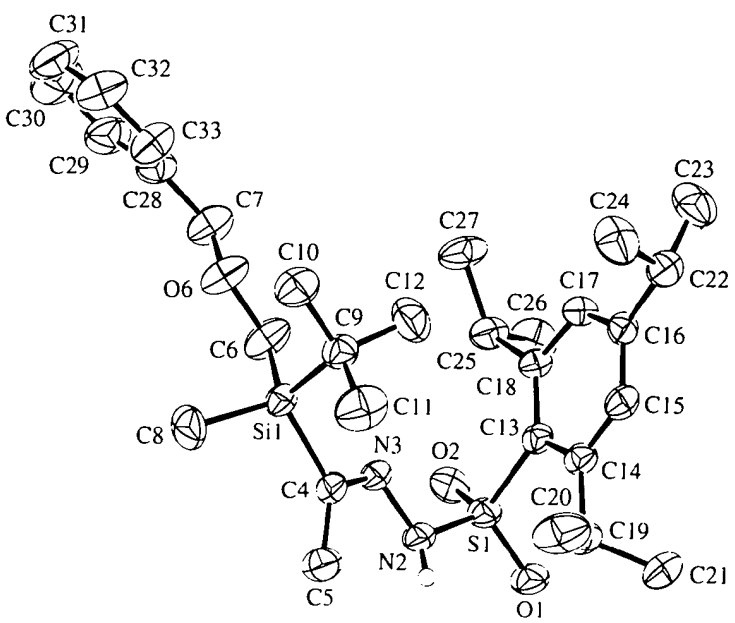

(a)

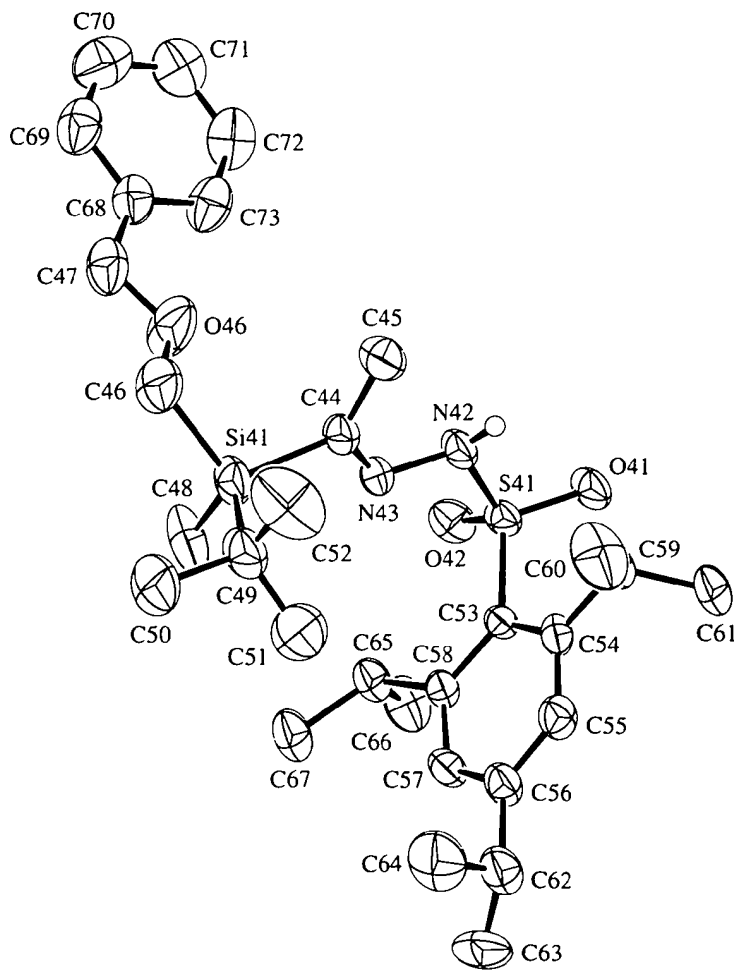

(b)

Fig. 1. Views of $(a)$ molecule $A$ and $(b)$ molecule $B$ showing the atom-labelling scheme. Displacement ellipsoids are drawn at the $40 \%$ probability level. Most of the $\mathrm{H}$ atoms have been omitted for clarity. 
molecules have similar bond lengths and angles. Except for atoms $\mathrm{O} 6, \mathrm{C} 7, \mathrm{C} 10, \mathrm{C} 11, \mathrm{C} 12$ and $\mathrm{C} 28-\mathrm{C} 33$, the non-H atoms in molecule $A$ are related by a pseudocentre of inversion to the corresponding atoms in molecule $B$, but additional crystallographic symmetry is not present.

The symmetry-independent molecules are linked into dimeric units by intermolecular hydrogen bonds (Table 2). The $\mathrm{N}-\mathrm{H}$ groups of molecules $A$ and $B$ act as donors, with one of the sulfonyl $\mathrm{O}$ atoms from molecules $B$ and $A$, respectively, acting as the acceptors.

\section{Experimental}

A solution of $56 \mathrm{mg}(0.21 \mathrm{mmol})$ of $(S)-(+)$-(I) (Bienz et al., $1995)$ and $66 \mathrm{mg}(0.22 \mathrm{mmol})$ of 2,4,6-triisopropylphenylsulfonylhydrazine in $5 \mathrm{ml}$ of dry acetonitrile was stirred at $296 \mathrm{~K}$ under nitrogen for $3 \mathrm{~h}$. The solvent was evaporated and the residue chromatographed $\left(\mathrm{SiO}_{2}\right.$, hexane/ethyl acetate $25: 1)$ to give $102 \mathrm{mg}(0.18 \mathrm{mmol}, 88 \%)$ of crystalline $(R)$ (+)-(II), which was recrystallized from 2-propanol (m.p. 364 $367 \mathrm{~K}) ;[\alpha]_{\mathrm{D}}^{23}+20^{\circ}$ (tetrahydrofuran, $\left.c 1.0\right)$. ' $\mathrm{H} \mathrm{NMR}\left(\mathrm{CDCl}_{3}\right.$, $300 \mathrm{MHz}$ ): $\delta$ (p.p.m.) 7.37-7.21 ( $m, 5 \mathrm{H}, 5 \times$ aromatic $\mathrm{CH}$ ), $7.13(s, 2 \mathrm{H}, 2 \times$ aromatic $\mathrm{CH}), 4.38\left(s, 2 \mathrm{H}, \mathrm{PhCH}_{2} \mathrm{O}\right)$, $4.18(h, J=6.8 \mathrm{~Hz}, 2 \mathrm{H}, 2 \times \mathrm{CH}), 3.27\left(s, 2 \mathrm{H}, \mathrm{SiCH}_{2} \mathrm{O}\right)$, $2.80(h, J=6.7 \mathrm{~Hz}, 1 \mathrm{H}, \mathrm{CH}), 1.79\left(s, 3 \mathrm{H}, \mathrm{CH}_{3}\right), 1.25-$ $1.22\left(m, 18 \mathrm{H}, 6 \times \mathrm{CH}_{3}\right), 0.69\left[s, 9 \mathrm{H}, \mathrm{C}\left(\mathrm{CH}_{3}\right)_{3}\right], 0.05$ $\left(s, 3 \mathrm{H}, \mathrm{SiCH}_{3}\right) ;{ }^{13} \mathrm{C} \mathrm{NMR}\left[\mathrm{CDCl}_{3}, 50.4 \mathrm{MHz}\right.$, multiplicities from 'distortionless enhancement by polarization transfer' experiments, atom numbering as in Fig. 1(a)]: $\delta$ (p.p.m.) 160.8 $(s, \mathrm{C} 4), 153.2(s, \mathrm{C} 13), 150.9(s, 2 \mathrm{C}, \mathrm{Cl} 4$ and $\mathrm{C} 18), 138.6(s$, $\mathrm{C} 16), 131.1(s, \mathrm{C} 18), 128.2(d, 2 \mathrm{C}, \mathrm{C} 29$ and $\mathrm{C} 33), 127.4(d$, $2 \mathrm{C}, \mathrm{C} 30$ and $\mathrm{C} 32), 127.3(d, \mathrm{C} 31), 123.5(d, 2 \mathrm{C}, \mathrm{C} 15$ and $\mathrm{C} 17), 77.1(t, \mathrm{C} 7), 59.9(t, \mathrm{C} 6), 34.2(d, \mathrm{C} 22), 29.7(d, 2 \mathrm{C}$, $\mathrm{C} 19$ and $\mathrm{C} 25), 26.5$ ( $q, 4 \mathrm{C}, \mathrm{C} 20, \mathrm{C} 21, \mathrm{C} 26$ and $\mathrm{C} 27), 24.7$ ( $q, 3 \mathrm{C}, \mathrm{C} 10, \mathrm{C} 11$ and $\mathrm{C} 12), 23.6(q, 2 \mathrm{C}, \mathrm{C} 23$ and $\mathrm{C} 24), 17.0$ $(s, \mathrm{C} 9), 19.3(q, \mathrm{C} 5),-9.5(q, \mathrm{C} 8)$; CI-MS $\left(\mathrm{NH}_{3}\right): \mathrm{m} / z 545$ $[M+1]^{+}$. Crystals suitable for the X-ray analysis were obtained by recrystallization from hexane with $5 \%$ methanol.

\section{Crystal data}

\section{$\mathrm{C}_{30} \mathrm{H}_{48} \mathrm{~N}_{2} \mathrm{O}_{3} \mathrm{SSi}$}

$M_{r}=544.87$

Triclinic

$P 1$

$$
\begin{aligned}
& a=10.890(1) \AA \\
& b=17.714(3) \AA \\
& c=8.940(1) \AA \\
& \alpha=97.24(1)^{\circ} \\
& \beta=105.824(8)^{\circ} \\
& \gamma=93.82(1)^{\circ} \\
& V=1636.8(4) \AA^{3} \\
& Z=2 \\
& D_{x}=1.106 \mathrm{Mg} \mathrm{m}^{-3}
\end{aligned}
$$

\section{Data collection}

Rigaku AFC-5R diffractometer

\author{
$\omega / 2 \theta$ scans \\ Absorption correction: \\ none \\ 13818 measured reflections \\ 13158 independent \\ reflections \\ 9678 observed reflections \\ $\left[F^{2}>2 \sigma\left(F^{2}\right)\right]$
}

\section{Refinement}

Refinement on $F^{2}$

$R(F)=0.0501$

$w^{\prime} R\left(F^{2}\right)=0.1446$

$S=1.012$

13157 reflections

698 parameters

$$
\begin{gathered}
w=1 /\left[\sigma^{2}\left(F_{o}^{2}\right)+(0.0712 P)^{2}\right. \\
+0.2780 P] \\
\text { where } P=\left(F_{o}^{2}+2 F_{r}^{2}\right) / 3 \\
(\Delta / \sigma)_{\max }=0.30
\end{gathered}
$$

\begin{tabular}{|c|c|c|c|c|c|}
\hline $\mathrm{H}), 4.38\left(s, 2 \mathrm{H}, \mathrm{PhCH}_{2} \mathrm{O}\right)$, & & $U_{\text {eq }}$ & /3) $\sum_{i} \Sigma_{j} U$ & ${ }_{j}^{*} \mathbf{a}_{i} \cdot \mathbf{a}_{j}$ & \\
\hline $\mathrm{CH}), 3.27\left(s, 2 \mathrm{H}, \mathrm{SiCH}_{2} \mathrm{O}\right)$, & & $x$ & $y$ & $z$ & $U_{\text {eq }}$ \\
\hline $1.79\left(s, 3 \mathrm{H}, \mathrm{CH}_{3}\right), 1.25-$ & S1 & $0.45900(7)$ & $0.94550(5)$ & $1.26160(9)$ & $0.0449(2)$ \\
\hline $69\left[\begin{array}{llllllll}5 & 9 \mathrm{H} & \mathrm{C}\left(\mathrm{CH}_{3}\right)_{3} & 0.05\end{array}\right.$ & Sil & $0.90769(9)$ & $0.84619(6)$ & $1.36163(12)$ & $0.0479(2)$ \\
\hline $09\left[S, 9 \mathrm{~A}, \mathcal{C}\left(\mathrm{CH}_{3}\right)_{3}\right]$, U.U丁 & Ol & $0.3988(2)$ & $1.00939(15)$ & $1.3153(3)$ & $0.0582(7)$ \\
\hline $\mathrm{l}_{3}, 50.4 \mathrm{MHz}$, multiplicities & $\mathrm{O} 2$ & $0.4267(3)$ & $0.9215(2)$ & $1.0974(3)$ & $0.0563(7)$ \\
\hline by polarization transfer' & O6 & $0.8970(3)$ & $0.72453(15)$ & $1.1429(3)$ & $0.0684(7)$ \\
\hline Fig. $1(a)]: \delta$ (p.p.m.) 160.8 & $\mathrm{~N} 2$ & $0.61 .32(.3)$ & $0.9744(2)$ & $1.3247(4)$ & $0.0482(7)$ \\
\hline$C 14$ and $C 18)$ & N3 & $0.6922(3)$ & $0.9159(2)$ & $1.3106(4)$ & $0.0459(7)$ \\
\hline+ and $(18), 150.0(5$ & Ct & $0.8129(4)$ & $0.9311(2)$ & $1.3789(4)$ & $0.0486(9)$ \\
\hline $2 \mathrm{C}, \mathrm{C} 29$ and $\mathrm{C} 33), 127.4(d$, & $\mathrm{C} 5$ & $0.8744(4)$ & $1.0088(2)$ & $1.4695(6)$ & $0.0684(12)$ \\
\hline 31$), 123.5(d, 2 \mathrm{C}, \mathrm{C} 15$ and & $\mathrm{C6}$ & $0.8247(5)$ & $0.7865(3)$ & $1.1677(5)$ & $0.0719(12)$ \\
\hline $34.2(d, \mathrm{C} 22), 29.7(d, 2 \mathrm{C}$ & $\mathrm{C7}$ & $0.8444(4)$ & $0.6754(2)$ & $1.0007(4)$ & $0.0695(11)$ \\
\hline 0 & $\mathrm{C} 8$ & $1.0756(4)$ & $0.8804(3)$ & $1.3664(7)$ & $0.0821(14)$ \\
\hline $\mathrm{C} 26$ and $(27), 24.7$ & $\mathrm{Cg}$ & $0.9102(4)$ & $0.7921(2)$ & $1.5320(5)$ & $0.0550(9)$ \\
\hline $5(q, 2 C, C 23$ and $C 24), 17.0$ & $\mathrm{C} 10$ & $1.0011(4)$ & $0.7293(3)$ & $1.5349(5)$ & $0.0726(12)$ \\
\hline $\mathrm{C} 8) ; \mathrm{CI}-\mathrm{MS}\left(\mathrm{NH}_{3}\right): \mathrm{m} / z 545$ & $\mathrm{Cll}$ & $0.9571(6)$ & $0.8469(3)$ & $1.6871(5)$ & $0.093(2)$ \\
\hline X-ray analysis were obtained & $\mathrm{C} 12$ & $0.7744(5)$ & $0.7544(3)$ & $1.5142(7)$ & $0.0859(15)$ \\
\hline & $\mathrm{C} 1.3$ & $0.4350(3)$ & $0.8666(2)$ & $1.3638(4)$ & $0.0395(7)$ \\
\hline methanol. & $\mathrm{C} 14$ & $0.4525(3)$ & $0.8828(2)$ & $1.5278(4)$ & $0.0447(8)$ \\
\hline & $C 15$ & $0.4324(4)$ & $0.8222(2)$ & $1.6057(4)$ & $0.0484(9)$ \\
\hline & $\mathrm{Cl} 6$ & $0.3959(4)$ & $0.7476(2)$ & $1.5298(4)$ & $0.0437(8)$ \\
\hline & $\mathrm{Cl}$ & $0.3805(4)$ & $0.7342(2)$ & $1.3710(4)$ & $0.0460(8)$ \\
\hline & $\mathrm{C} 18$ & $0.4010(3)$ & $0.7912(2)$ & $1.2827(4)$ & $0.0414(8)$ \\
\hline Mo $K \alpha$ radiation & $\mathrm{C} 19$ & $0.4982(4)$ & $0.9617(2)$ & $1.631 .3(5)$ & $0.0566(10)$ \\
\hline$\lambda=0.71073 \AA$ & $\mathrm{C} 20$ & $0.6186(5)$ & $0.9577(3)$ & $1.7623(7)$ & $0.097(2)$ \\
\hline Cell parameters from 25 & $\mathrm{C} 21$ & $0.3910(5)$ & $0.9886(3)$ & $1.7010(6)$ & $0.0730(13)$ \\
\hline AIICECIS MUII LJ & $\mathrm{C} 22$ & $0.3852(4)$ & $0.6829(2)$ & $1.6248(5)$ & $0.0569(10)$ \\
\hline reflections & $\mathrm{C} 23$ & $0.2886(5)$ & $0.6152(3)$ & $1.5385(6)$ & $0.0847(15)$ \\
\hline$\theta=18-20^{\circ}$ & $\mathrm{C} 24$ & $0.5154(5)$ & $0.6570(3)$ & $1.6878(7)$ & $0.088(2)$ \\
\hline$\mu=0.165 \mathrm{~mm}^{-1}$ & $\mathrm{C} 25$ & $0.3841(4)$ & $0.7650(2)$ & $1.1077(4)$ & $0.0546(10)$ \\
\hline$T=243(1) \mathrm{K}$ & $\mathrm{C} 26$ & $0.2446(5)$ & $0.7694(3)$ & $1.0134(5)$ & $0.0730(12)$ \\
\hline Prism & $\mathrm{C} 27$ & $0.4248(6)$ & $0.6846(3)$ & $1.0759(5)$ & $0.0796(15)$ \\
\hline & $\mathrm{C} 28$ & $0.9371(4)$ & $0.6192(2)$ & $0.9765(4)$ & $0.0599(10)$ \\
\hline $0.38 \times 0.35 \times 0.35 \mathrm{~mm}$ & $\mathrm{C} 29$ & $0.9390(6)$ & $0.5915(3)$ & $0.8258(5)$ & $0.0789(14)$ \\
\hline Colourless & C.30 & $1.0253(8)$ & $0.5405(4)$ & $0.8024(7)$ & $0.113(2)$ \\
\hline & C 31 & $1.1092(6)$ & $0.5177(3)$ & $0.9269(8)$ & $0.103(2)$ \\
\hline & C.32 & $1.1078(6)$ & $0.5443(3)$ & $1.0754(7)$ & $0.092(2)$ \\
\hline & $\mathrm{C} 33$ & $1.0227(5)$ & $0.5944(3)$ & $1.1006(5)$ & $0.0739(12)$ \\
\hline & $S 41$ & $0.60242(7)$ & $0.19011(5)$ & $0.58597(9)$ & $0.0464(2)$ \\
\hline & $\mathrm{Si} 41$ & $0.16258(11)$ & $0.28851(7)$ & $0.53322(15)$ & $0.0656(3)$ \\
\hline & 041 & $0.6629(2)$ & $0.12783(15)$ & $0.5256(3)$ & $0.0594(7)$ \\
\hline & $\mathrm{O} 42$ & $0.6408(3)$ & $0.2129(2)$ & $0.7527(3)$ & $0.0609(7)$ \\
\hline & 046 & $0.0545(3)$ & $0.1987(2)$ & $0.6862(5)$ & $0.1082(12)$ \\
\hline$R_{\mathrm{int}}=0.1165$ & N42 & $0.4492(3)$ & $0.1596(2)$ & $0.5278(4)$ & $0.0524(8)$ \\
\hline$\theta_{\max }=27.5^{\circ}$ & $\mathrm{N} 43$ & $0.3721(3)$ & $0.2176(2)$ & $0.5518(4)$ & $0.0507(8)$ \\
\hline
\end{tabular}

$$
\begin{aligned}
& h=-11 \rightarrow 14 \\
& k=-22 \rightarrow 22 \\
& l=-11 \rightarrow 11 \\
& 3 \text { standard reflections } \\
& \text { monitored every } 150 \\
& \text { reflections } \\
& \text { intensity decay: } \\
& \text { insignificant }
\end{aligned}
$$

$$
\begin{aligned}
& \Delta \rho_{\max }=0.23{\mathrm{e} \AA^{-3}}^{-3} \rho_{\min }=-0.32 \AA^{-3}
\end{aligned}
$$

Atomic scattering factors from International Tables for Crystallography (1992, Vol. C, Tables 4.2.6.8 and 6.1.1.4)

Absolute configuration: Flack (1983) parameter $=-0.02(7)$

Table 1. Fractional atomic coordinates and equivalent isotropic displacement parameters $\left(\AA^{2}\right)$ 


$\begin{array}{lrlll}\text { C44 } & 0.2508(4) & 0.2038(2) & 0.4912(5) & 0.0522(9) \\ \text { C45 } & 0.1861(4) & 0.1291(3) & 0.3945(7) & 0.0764(14) \\ \text { C46 } & 0.0127(5) & 0.2502(3) & 0.5772(7) & 0.0891(15) \\ \text { C47 } & -0.0263(4) & 0.1819(3) & 0.7739(6) & 0.0792(12) \\ \text { C48 } & 0.2637(6) & 0.3511(4) & 0.7144(7) & 0.106(2) \\ \text { C49 } & 0.1279(5) & 0.3406(3) & 0.3616(6) & 0.0802(14) \\ \text { C50 } & 0.0448(7) & 0.4064(4) & 0.3875(9) & 0.119(2) \\ \text { C51 } & 0.2526(6) & 0.3757(4) & 0.3392(9) & 0.120(2) \\ \text { C52 } & 0.0511(8) & 0.2856(4) & 0.2100(7) & 0.139(3) \\ \text { C53 } & 0.6208(3) & 0.2716(2) & 0.4889(4) & 0.0406(7) \\ \text { C54 } & 0.6045(3) & 0.2582(2) & 0.3260(4) & 0.0468(9) \\ \text { C55 } & 0.6243(4) & 0.3212(2) & 0.2527(4) & 0.0561(10) \\ \text { C56 } & 0.6524(4) & 0.3957(2) & 0.3320(5) & 0.0568(10) \\ \text { C57 } & 0.6639(4) & 0.4064(2) & 0.4910(4) & 0.0535(9) \\ \text { C58 } & 0.6469(4) & 0.3478(2) & 0.5738(4) & 0.0434(8) \\ \text { C59 } & 0.5666(4) & 0.1810(2) & 0.2200(5) & 0.0566(10) \\ \text { C60 } & 0.4448(5) & 0.1835(4) & 0.0836(7) & 0.110(2) \\ \text { C61 } & 0.6751(5) & 0.1551(3) & 0.1547(6) & 0.0782(14) \\ \text { C62 } & 0.6613(6) & 0.4627(3) & 0.2432(5) & 0.0759(14) \\ \text { C63 } & 0.7582(7) & 0.5299(3) & 0.3373(8) & 0.102(2) \\ \text { C64 } & 0.5255(7) & 0.4864(4) & 0.1821(8) & 0.108(2) \\ \text { C65 } & 0.6541(4) & 0.3704(2) & 0.7483(4) & 0.0523(9) \\ \text { C66 } & 0.7884(5) & 0.3706(3) & 0.8531(5) & 0.0750(13) \\ \text { C67 } & 0.6057(5) & 0.4489(3) & 0.7781(5) & 0.0683(12) \\ \text { C68 } & 0.0125(4) & 0.1176(3) & 0.8614(5) & 0.0642(10) \\ \text { C69 } & -0.0695(5) & 0.0862(3) & 0.9392(6) & 0.0807(14) \\ \text { C70 } & -0.0387(6) & 0.0266(4) & 1.0169(6) & 0.096(2) \\ \text { C71 } & 0.0747(6) & -0.0054(4) & 1.0201(6) & 0.091(2) \\ \text { C72 } & 0.1557(5) & 0.0246(3) & 0.9464(5) & 0.0831(14) \\ \text { C73 } & 0.1260(4) & 0.0855(3) & 0.8670(5) & 0.0764(13) \\ & & & & \end{array}$

positions and allowed to ride on their respective parent $C$ atom, with fixed isotropic displacement parameters equal to $1.2 U_{\mathrm{eq}}$ of the parent $\mathrm{C}$ atom ( $1.5 U_{\mathrm{eq}}$ for methyl groups). The origin was fixed by using the least-squares-restraints method of Flack \& Schwarzenbach (1988). For the determination of the absolute configuration, the TWIN and BASF options of SHELXL93 (Sheldrick, 1993) were used in the final refinement to refine the absolute-structure parameter (Flack, 1983). The refined value confidently confirms that the atomic coordinates represent the correct enantiomorph. A refinement of the structure using the centrosymmetric space group $P \overline{1}$ showed that many of the non- $\mathrm{H}$ atoms of molecule $A$ are almost perfectly related by a centre of inversion to the corresponding atoms in molecule $B$. However, atoms $\mathrm{O} 6, \mathrm{C} 7, \mathrm{C} 10, \mathrm{C} 11, \mathrm{C} 12$ and $\mathrm{C} 28-\mathrm{C} 33$ can only be refined in $P \overline{1}$ if they are included as disordered atoms, whereas the structure could be refined without disorder in the non-centrosymmetric space group $P 1$. The presence of a racemic crystal and additional crystallographic symmetry can therefore be discounted.

MSCIAFC Diffractometer Control Software (Molecular Structure Corporation, 1991) was used for data collection and cell determination, and TEXSAN (Molecular Structure Corporation, 1989) was used for data reduction. The structure was solved by direct methods using SHELXS86 (Sheldrick, 1990) and refined with SHELXL93. The molecular graphics were produced using ORTEPII (Johnson, 1976).

Table 2. Selected geometric parameters $\left(\AA^{\circ},{ }^{\circ}\right)$

\begin{tabular}{|c|c|c|c|}
\hline $\mathrm{SI}-\mathrm{Ol}$ & $1.440(3)$ & $\mathrm{S} 41-\mathrm{O} 41$ & $1.444(3)$ \\
\hline $\mathrm{S} 1-\mathrm{O} 2$ & $1.414(3)$ & $\mathrm{S} 4 \mathrm{I}-\mathrm{O} 42$ & $1.430(3)$ \\
\hline $\mathrm{S} 1-\mathrm{N} 2$ & $1.642(3)$ & $\mathrm{S} 41-\mathrm{N} 42$ & $1.638(3)$ \\
\hline $\mathrm{SI}-\mathrm{C} 13$ & $1.806(3)$ & $\mathrm{S} 41-\mathrm{C} 53$ & $1.802(3)$ \\
\hline $\mathrm{Sil}-\mathrm{C} 4$ & $1.895(4)$ & $\mathrm{Si} 41-\mathrm{C} 44$ & $1.888(4)$ \\
\hline $\mathrm{Si} 1-\mathrm{C} 6$ & $1.870(5)$ & $\mathrm{Si} 4 \mathrm{I}-\mathrm{C} 46$ & $1.884(5)$ \\
\hline $\mathrm{Sil}-\mathrm{C} 8$ & $1.874(4)$ & $\mathrm{Si} 41-\mathrm{C} 48$ & $1.865(6)$ \\
\hline $\mathrm{Sil}-\mathrm{C} 9$ & $1.895(4)$ & $\mathrm{Si} 4 \mathrm{l}-\mathrm{C} 49$ & $1.853(5)$ \\
\hline $\mathrm{O} 6-\mathrm{C} 6$ & $1.422(5)$ & $\mathrm{O} 46-\mathrm{C} 46$ & $1.422(6)$ \\
\hline $\mathrm{O} 6-\mathrm{C} 7$ & $1.399(4)$ & $\mathrm{O} 46-\mathrm{C} 47$ & $1.370(5)$ \\
\hline $\mathrm{N} 2-\mathrm{N} 3$ & $1.406(4)$ & $\mathrm{N} 42-\mathrm{N} 43$ & $1.401(4)$ \\
\hline $\mathrm{N} 3-\mathrm{C} 4$ & $1.283(5)$ & $\mathrm{N} 43-\mathrm{C} 44$ & $1.277(5)$ \\
\hline $\mathrm{C} 4-\mathrm{C} 5$ & $1.516(6)$ & $\mathrm{C} 44-\mathrm{C} 45$ & $1.501(6)$ \\
\hline $\mathrm{C} 7-\mathrm{C} 28$ & $1.503(5)$ & $\mathrm{C} 47-\mathrm{C} .68$ & $1.482(6)$ \\
\hline $\mathrm{O} 1-\mathrm{SI}-\mathrm{O} 2$ & $117.1(2)$ & $\mathrm{O} 41-\mathrm{S} 41-\mathrm{O} 42$ & $116.9(2)$ \\
\hline $\mathrm{N} 2-\mathrm{SI}-\mathrm{C} 13$ & $105.9(2)$ & $\mathrm{N} 42-\mathrm{S} 41-\mathrm{C} 5 \mathrm{i}$ & $106.2(2)$ \\
\hline $\mathrm{C} 4-\mathrm{Sil}-\mathrm{C} 6$ & $106.6(2)$ & $\mathrm{C} 44-\mathrm{Si} 41-\mathrm{C} 46$ & $107.1(2)$ \\
\hline $\mathrm{C} 4-\mathrm{Sil}-\mathrm{C} 8$ & $109.7(2)$ & $\mathrm{C} 44-\mathrm{Si} 41-\mathrm{C} 48$ & $108.7(2)$ \\
\hline $\mathrm{C} 4-\mathrm{Sil}-\mathrm{C} 9$ & $108.6(2)$ & $\mathrm{C} 44-\mathrm{Si} 41-\mathrm{C} 49$ & $109.2(2)$ \\
\hline $\mathrm{C} 6-\mathrm{Sil}-\mathrm{C} 8$ & $109.8(.3)$ & $\mathrm{C} 46-\mathrm{Si} 41-\mathrm{C} 48$ & $107.5(3)$ \\
\hline $\mathrm{C} 6-\mathrm{Sil}-\mathrm{C} 9$ & $112.0(2)$ & $\mathrm{C} 46-\mathrm{Si} 41-\mathrm{C} 49$ & $112.8(2)$ \\
\hline $\mathrm{C} 8-\mathrm{Sil}-\mathrm{C} 9$ & $110.1(2)$ & $\mathrm{C} 48-\mathrm{Si} 41-\mathrm{C} 49$ & $111.5(3)$ \\
\hline $\mathrm{C} 6-\mathrm{O} 6-\mathrm{C} 7$ & $114.6(3)$ & $\mathrm{C} 46-\mathrm{O} 46-\mathrm{C} 47$ & $116.0(3)$ \\
\hline $\mathrm{S} 1-\mathrm{N} 2-\mathrm{N} 3$ & $113.9(2)$ & $\mathrm{S} 41-\mathrm{N} 42-\mathrm{N} 43$ & $112.5(3)$ \\
\hline $\mathrm{N} 2-\mathrm{N} 3-\mathrm{C} 4$ & $117.6(3)$ & $\mathrm{N} 42-\mathrm{N} 43-\mathrm{C} 44$ & $118.3(3)$ \\
\hline $\mathrm{Sil}-\mathrm{C} 4-\mathrm{N} 3$ & $113.6(3)$ & $\mathrm{Si} 41-\mathrm{C} 44-\mathrm{N} 43$ & $112.5(3)$ \\
\hline $\mathrm{Sil}-\mathrm{C} 4-\mathrm{C} 5$ & $122.7(3)$ & $\mathrm{Si} 41-\mathrm{C} 44-\mathrm{C} 45$ & $123.8(3)$ \\
\hline $\mathrm{N} 3-\mathrm{C} 4-\mathrm{C} 5$ & $123.7(3)$ & $\mathrm{N} 43-\mathrm{C} 44-\mathrm{C} 45$ & $12.3 .7(4)$ \\
\hline $\mathrm{Sil}-\mathrm{C} 6-\mathrm{O} 6$ & $109.1(3)$ & $\mathrm{Si} 41-\mathrm{C} 46-\mathrm{O} 46$ & $105.1(3)$ \\
\hline $\mathrm{O} 6-\mathrm{C} 7-\mathrm{C} 28$ & $109.9(3)$ & $\mathrm{O} 46-\mathrm{C} 47-\mathrm{C} 68$ & $112.0(4)$ \\
\hline$D \multimap \mathrm{H} \cdots A$ & $D-\mathrm{H}$ & $D \cdots A$ & $D \multimap \mathrm{H} \cdots A$ \\
\hline $\mathrm{N} 2-\mathrm{H} 2 \ldots \mathrm{O} 41^{\prime}$ & $0.88(3)$ & $2.991(4)$ & $162(2)$ \\
\hline $\mathrm{N} 42-\mathrm{H} 42 \ldots \mathrm{O} 1^{\prime \prime}$ & $0.89(2)$ & $2.993(4)$ & $148(2)$ \\
\hline
\end{tabular}

Symmetry codes: (i) $x, 1+y, 1+z$; (ii) $x, y-1, z-1$.

The data collection was extended to include the measurement of the intensities of the Friedel opposites of those reflections in the unique hemisphere for which $\theta<25^{\circ}$. The amine $\mathrm{H}$ atoms were located in a difference electron density map and refined isotropically with bond-length restraints of 0.90 (1) $\AA$. All other $\mathrm{H}$ atoms were placed in geometrically calculated

Professor Dr M. Hesse, University of Zürich, is thanked for providing us with equipment, laboratory space and regular occasions for professional discussions.

Lists of structure factors, anisotropic displacement parameters, $\mathrm{H}$ atom coordinates and complete geometry have been deposited with the IUCr (Reference: AB1320). Copies may be obtained through The Managing Editor, International Union of Crystallography, 5 Abbey Square, Chester $\mathrm{CHI} 2 \mathrm{HU}$, England.

\section{References}

Bienz, S., Bratovanov, S., Chapeaurouge, A., Huber, P., Pietzsch, M. \& Syldatk, C. (1995). Proceedings of the 3rd International Symposium on Biochemical Engineering, Stuttgart, Germany. In the press.

Bienz, S. \& Chapeaurouge, A. (1991). Helv. Chim. Acta, 74, 14771488.

Chapeaurouge, A. \& Bienz, S. (1993). Helv. Chim. Acta, 76, 18761889.

Enev, V., Stojanova, D. \& Bienz, S. (1995). Unpublished results.

Flack, H. D. (1983). Acta Cryst. A39, 876-881.

Flack, H. D. \& Schwarzenbach, D. (1988). Acta Cryst. A44, 499-506.

Johnson, C. K. (1976). ORTEPII. Report ORNL-5138. Oak Ridge National Laboratory, Tennessee, USA.

Molecular Structure Corporation (1989). TEXSAN. Single Crystal Structure Analysis Software. MSC, 3200 Research Forest Drive, The Woodlands, TX 77381, USA.

Molecular Structure Corporation (1991). MSC/AFC Diffractometer Control Software. MSC, 3200 Research Forest Drive, The Woodlands, TX 77381, USA.

Sheldrick, G. M. (1990). SHELXS86. Acta Cryst. A46, 467-473.

Sheldrick, G. M. (1993). SHELXL93. Program for the Refinement of Crystal Structures. University of Göttingen, Germany. 